\title{
Fuzzy Adaptation Algorithms' Control for Robot Manipulators with Uncertainty Modelling Errors
}

\author{
Yongqing Fan $\left(\mathbb{D},{ }^{1}\right.$ Keyi Xing, ${ }^{1}$ and Xiangkui Jiang $\mathbb{D}^{2}$ \\ ${ }^{1}$ The State Key Laboratory for Manufacturing Systems Engineering and Systems Engineering Institute, Xi'an Jiaotong University, \\ Xian 710049, China \\ ${ }^{2}$ The School of Automation, Xian University of Posts and Telecommunications, Xian, China
}

Correspondence should be addressed to Yongqing Fan; yongq-f@163.com

Received 19 February 2018; Accepted 27 March 2018; Published 10 May 2018

Academic Editor: Jing $\mathrm{Na}$

Copyright (C) 2018 Yongqing Fan et al. This is an open access article distributed under the Creative Commons Attribution License, which permits unrestricted use, distribution, and reproduction in any medium, provided the original work is properly cited.

\begin{abstract}
A novel fuzzy control scheme with adaptation algorithms is developed for robot manipulators' system. At the beginning, one adjustable parameter is introduced in the fuzzy logic system, the robot manipulators system with uncertain nonlinear terms as the master device and a reference model dynamic system as the slave robot system. To overcome the limitations such as online learning computation burden and logic structure in conventional fuzzy logic systems, a parameter should be used in fuzzy logic system, which composes fuzzy logic system with updated parameter laws, and can be formed for a new fashioned adaptation algorithms controller. The error closed-loop dynamical system can be stabilized based on Lyapunov analysis, the number of online learning computation burdens can be reduced greatly, and the different kinds of fuzzy logic systems with fuzzy rules or without any fuzzy rules are also suited. Finally, effectiveness of the proposed approach has been shown in simulation example.
\end{abstract}

\section{Introduction}

Technologies of learning and adaptation in complex dynamical systems have been become an indispensable part of modern high-tech and attracted the attention in different range of engineering fields. More recently, much effort has been made to handle applications in medical researches [1], virtual reality [2], teleoperation and entertainment [3], and so on. In robotic systems, many technologies about dynamical complex systems can be described as learning human-like skills, such as making the states following a given reference model in [4]; the robot slave arm is employed to drive the adaptation algorithms according to human tutors behavior [5]. Usually, the motion of a master device is controlled by human operator controls, and the master device generates commanding trajectories that are passed to slave systems in order to execute the task by the communication channels [6]. Many kinds of learning and adaptation algorithms control strategies for teleoperated robot systems have been reported in order to improve the controllability and reliability in $[7,8]$. Based on wave variables and corrections, a novel control scheme is established to deal with the stability of the closed-loop systems in [9]. From the viewpoint of mathematics, ordinary differential equations (ODE) can be represented between the master and slave of robot systems, so the synchronization between them is closely similar to observer problem $[10,11]$, which means that some mathematical methods in control theory may be employed to solve the problem of synchronization for master-slave robot systems. A novel shared control system with task motion and self-motion is to achieve accurate object manipulation for Baxter robot manipulator by users mind in [12]. In order to ensure robustness and realization capability of the controllers, disturbance observer is designed by using linear matrix inequality (LMI) in [13].

Generally speaking, it is difficult to research on controlling system with unknown parameters or uncertainties models [14, 15], and hence how to control complexity systems such as robot manipulator systems with uncertain terms becomes a topic deserving of study. Adaptive learning algorithm of parameter estimation is utilized to control robot arm systems with unknown parameters [16]. In [17], an extended state observer is designed to estimate the unknown friction and disturbances in systems such that tracking performance is 
achieved. For the problem of unknown model of vehicle engine torque, unknown input observer and adaptive parameters estimation are proposed in [18]. Many other results have shown that neural network (NN) [19-23] and fuzzy logic systems (FLSs) are universal approximators [24-26] with online learning capability to emulate complicated nonlinearity and uncertain functions. For an uncertain three-link flexiblejoint electrically driven manipulator with complex nonlinear functions, an adaptation algorithms observer combining with $\mathrm{NNs}$ is used to estimate the uncertain information such as the link and actuator velocity in systems in [27]; a link position tracking controller with estimated states is proposed. A novel control is investigated in [28], incorporated with NNs and adaptation algorithms technology; the output feedback controller with an observer is built to stabilize the closedloop systems, and the $\mathrm{NN}$ is to satisfy or improve some performance of robot dynamical systems. In order to extend the semiglobal stability to global stability by using NNs controller, a switching mechanism in [29] is formatted for the control task of bimanual robots systems. An augmented NN combining with prescribed performance function is utilized to compensate for unknown active vehicle suspension systems in [30], which can ensure that the transient and steadystate suspension response is guaranteed. Because the extreme learning machine (ELM) has many advantages compared to the traditional neural network, such as convenience for implementation, the fast training speed, and the least manual intervention, [31] brought a novel ELM control to tackle the problems of uncertain robot manipulators. In [32], an amplitude limited controller was employed to active compensation for unknown parameters effects in dynamical systems, and the controller designed can guarantee semiglobal asymptotic stability of the task-space errors. Because FLSs have high interpretability of rich expert experience comprised with NNs, many researchers incline to deal with uncertainties terms in systems by using FLSs combining with the adaptation algorithms technology. From a mathematical point of view, the fuzzy adaptation algorithms control ideas in [24, $25,32]$ depend on the output of fuzzy logic systems, mainly represented as a linear basis function, and the combinatorial coefficients can be adjusted automatically online. On one hand, many fuzzy rules must be used in order to improve the accuracy of approximation, so great rules are used for this intent. But, on the other hand, too many fuzzy rules will make much more burdens of online learning computation, and time delay must be required; these phenomena can destroy or violate the stability and performance index of the robots dynamical systems.

As an attempt to solve the above discussed problem, the bounded norm about the optimal fuzzy parameter vector is assumed in $[33,34]$, with which the amount of online learning updated laws is decreased to only one. However, these design methods induced another trouble that the number of the input variables increases greatly with the increase of dimension in systems. Besides, the parameter of bounded norm is assumed to be known previously in these references. It should be also noted that the above fuzzy adaptation algorithms methods can not be used in different forms of FLSs, such as FLSs without if-then rules, or the outputs of
FLSs can not be written as the linear functions [35, 36]. So it is noteworthy to exploit another design adaptation algorithm that can be utilized in the FLSs with linear combinatorial parameters or a great variety of the outputs forms of FLSs. In order to achieve this purpose, we consider the approximation accuracies of FLSs, utilized to be parameter estimated online instead of online estimation of the linear combination coefficients or the ideal norm of weighted vectors. The advantage of this design is that the adaptive online learning laws only focus on the approximation accuracies. However, only the index of approximation accuracy is not enough to synthesize the adaptation algorithms fuzzy controller. Therefore, other parameters independent of the structure of FLSs are necessary to be considered. For this objective, a time-varying parameter is introduced in FLSs, and a design method is used in the master robot system which guarantees good tracking effect compared with the conventional fuzzy adaptation algorithms controller.

This paper puts forward a fuzzy logic system with timevarying parameter, which is proposed to design adaptation algorithms controller for the problem of synchronization between the robot manipulators dynamical system with unknown nonlinear terms and reference model system; we design the fuzzy adaptation algorithms controller based on sliding surface and Lyapunov stabilized theory analysis. In Section 2, some assumptions and descriptions of dynamical system are given. FLS based on linguistics is introduced in Section 3. An adaptation algorithm control is designed in Section 4. Simulation example is performed to test the designed controller, and conclusion is given in the end.

\section{System Description and Assumptions}

The dynamic equation of the robot manipulators can be represented as follows:

$$
M(q) \ddot{q}+C(q, \dot{q}) \dot{q}+G(q)+F_{d} \dot{q}+F_{s}(q)+T_{d}=\tau,
$$

where $q(t)$ is the $n \times 1$ vector of joint angular positions. $M(q) \in R^{n \times n}, C(q, \dot{q}) \dot{q} \in R^{n}, G(q) \in R^{n}$ are the inertial matrix, Coriolis and centrifugal matrix, and gravity vector, respectively. $F_{d} \dot{q} \in R^{n}$ is viscous friction, $F_{s}(q) \in R^{n}$ denote a positive definite matrix with bounded constant satisfying $\left\|F_{s}(q)\right\| \leq \tau_{s}$, and $\tau_{s}$ is a known constant. $T_{d} \in R^{n}$ is continuous external disturbance, which satisfied $\left\|T_{d}\right\| \leq \tau_{d}$. $\tau \in R^{n \times 1}$ denotes vector of applied joint torques and will be designed.

Assumption 1. Matrix vector $G(q)=\left[g_{1}(q), \ldots, g_{n}(q)\right]^{T}$ is uniformly bounded and uniformly continuous if $g_{i}(q)(i=$ $1,2, \ldots, n)$ is uniformly bounded and continuous and satisfies $\left|g_{i}(q)\right| \leq \tau_{g}^{i}$, and $\tau_{g}^{i}$ is a known positive scalar.

From Assumption 1, we denote $\tau_{g}=\max \left\{\tau_{g}^{1}, \ldots, \tau_{g}^{n}\right\}$ and obtain $\|G(q)\| \leq \tau_{g}$.

Assumption 2. Function vector $F_{d} \dot{q}=\left[\Delta_{1}, \ldots, \Delta_{n}\right]^{T}$ is unknown on the compact set $\widetilde{V}$, and $\Delta_{i}$ satisfies Lipschitz 
condition. That is to say, there exists Lipschitz coefficient $l_{i}$ satisfying $\left|\Delta_{i}\left(z_{1}\right)-\Delta_{i}\left(z_{2}\right)\right| \leq l_{i}\left|z_{1}-z_{2}\right|$.

Property 3. Matrix $M(q)$ is a known positive symmetric matrix, the inverse matrix $M^{-1}(q)$ exists, satisfying $M_{\min }^{-1} \leq$ $\left\|M^{-1}(q)\right\| \leq M_{\max }^{-1}$, and $M_{\min }^{-1}$ and $M_{\max }^{-1}$ are two known positive constants.

If we define state vector $x=\left[q^{T}, \dot{q}^{T}\right]^{T}$, dynamic system (1) can be rewritten as

$$
\begin{aligned}
& \dot{x} \\
& =\left[\begin{array}{c}
\dot{q} \\
-M^{-1}(q)\left[C(q, \dot{q}) \dot{q}+G(q)+F_{d} \dot{q}+F_{s}(q)+T_{d}\right]
\end{array}\right] \\
& \quad+\left[\begin{array}{l}
O \\
I
\end{array}\right]+M^{-1}(q) \tau,
\end{aligned}
$$

where $O \in R^{n \times n}$ is a matrix with all elements 0 , and $I \in$ $R^{n \times n}$ denotes identity matrix. The following desired reference model for the plant can be represented as in [37]:

$$
\left[\begin{array}{l}
\dot{q}_{m} \\
\ddot{q}_{m}
\end{array}\right]=\left[\begin{array}{cc}
O & I \\
\bar{P} & \bar{Q}
\end{array}\right]\left[\begin{array}{l}
q_{m} \\
\dot{q}_{m}
\end{array}\right]+\left[\begin{array}{c}
O \\
B_{1}
\end{array}\right] r \triangleq A_{m} x_{m}+B_{m} r,
$$

where $\bar{P}=\operatorname{diag}\left\{p_{1}, \ldots, p_{n}\right\}, \bar{Q}=\operatorname{diag}\left\{q_{1}, \ldots, q_{n}\right\}$, and $B_{1}=$ $\operatorname{diag}\left\{b_{1}, \ldots, b_{n}\right\}$ are all constant matrices, $p_{i}, q_{i}$, and $b_{i}(1 \leq$ $i \leq n$ ) are chosen such that the reference model (3) can be stable. In this paper, vectors $r, q_{m}$, and $\dot{q}_{m}$ are assumed to be measurable.

Defining $\varepsilon=q-q_{r}, e=\left[\varepsilon^{T}, \dot{\varepsilon}^{T}\right]^{T}$, then we get the error dynamical system as

$$
\dot{e}=A_{m} e+B h(q, \dot{q}, \tau, r),
$$

where $h(q, \dot{q}, \tau, r)=M^{-1}(q) \tau-B_{1} r-\bar{P} q-\bar{Q} \dot{q}-$ $M^{-1}(q)\left[C(q, \dot{q}) \dot{q}+G(q)+F_{d} \dot{q}+F_{s}(q)+T_{d}\right]$ and $B=[O, I]^{T}$.

Assumption 4. The matrix pair $\left(A_{m}, B\right)$ is completely controllable; it is means that, for any given matrix $Q>0$, there must exist a matrix $K \in R^{m \times n}$ such that the Lyapunov equation as follows has only one positive definition symmetry matrix solution $P$ :

$$
\left(A_{m}+B K\right)^{T} P+P\left(A_{m}+B K\right)+Q=0 .
$$

The control gain matrix $K$ can be obtained by solving linear matrix inequality (LMI) $X A_{m}^{T}+A_{m} X+Y^{T} B^{T}+B Y \leq-\widetilde{Q}$ for any positive matrix $\widetilde{Q}>0$ and matrices $K=Y X^{-1}$, $X=P^{-1}$, and $Y=K X$.

\section{Description of Fuzzy Logic Systems}

In this section, the Mamdani type FLS with $q$ fuzzy rules and the $l$ th rule is to be considered as the following format:

$$
\begin{aligned}
& \text { If } x_{1} \text { is } A_{1}^{l} \text { and } x_{2} \text { is } A_{2}^{l} \text { and } \cdots \text { and } x_{n} \text { is } A_{n}^{l} \text {, } \\
& \text { then } y^{l} \text { is } B^{l}, \quad l=1,2, \ldots, q \text {, }
\end{aligned}
$$

where $A_{s}^{l}$ and $B^{l}$ represent fuzzy sets of variable linguistics; $\mu_{A_{s}^{l}}(s=1,2, \ldots, n)$ and $\mu_{B^{l}}$ denote the membership function, respectively.

According to the reference in [26], then the output of fuzzy logic system (6) can be written by the following form:

$$
y=F(y)=\frac{\sum_{l=1}^{q} y^{l} \prod_{s=1}^{n} A_{s}^{l}\left(x_{s}\right)}{\sum_{l=1}^{q} \prod_{s=1}^{n} A_{s}^{l}\left(x_{s}\right)},
$$

where $y^{l}=\max _{y \in R} \mu_{B^{l}}\left(y^{l}\right)$. Now, a nonzero time-varying parameter $\rho=\rho(t)$ is introduced in (7); then we can get the following result:

$$
\bar{y}=F(\bar{y})=\frac{\sum_{l=1}^{q} y^{l} \prod_{s=1}^{n} A_{s}^{l}\left(x_{s} / \rho\right)}{\sum_{l=1}^{q} \prod_{s=1}^{n} A_{s}^{l}\left(x_{s} / \rho\right)} .
$$

Remark 5. The work mechanism of the time-varying $\rho$ is to make the input signal enter $\|x\| \leq \alpha|\rho|$; the final output of the FLS is obtained as the form of (8); then the approximation accuracy can be online updated by parameter $\rho$.

Lemma 6. If the uncertain continuous nonlinear functions $\xi_{i}(z) \in R^{n}$ satisfy Lipschitz condition with coefficients $\theta_{i}$ and FLS $F_{i}$ exists, making $\sup _{z \in \widetilde{U}}\left|\theta_{i}(z)-F_{i}(z)\right| \leq N_{i}$, where $\widetilde{U}=$ $\{z|\|z\| \leq| \rho \mid \alpha\}$, then the following inequality holds:

$$
\sup _{\|z\| \leq|\rho| \alpha}\left|\xi_{i}(z)-F_{i}\left(\frac{z}{\rho}\right)\right| \leq \theta_{i} \alpha|\rho-1|+N_{i} .
$$

Proof. Since $\xi_{i}(z)$ satisfies Lipschitz condition with coefficient $\theta_{i}$, then $\left|\xi_{i}(z)-\xi_{i}(z / \rho)\right| \leq \theta_{i}\|z-z / \rho\|$. If $z \in\{z|\|z\| \leq| \rho \mid \alpha\}$ is true, we have the following result:

$$
\begin{aligned}
\left|\xi_{i}(z)-F_{i}\left(\frac{z}{\rho}\right)\right| \leq & \left|\xi_{i}(z)-\xi_{i}\left(\frac{z}{\rho}\right)\right| \\
& +\left|\xi_{i}\left(\frac{z}{\rho}\right)-F_{i}\left(\frac{z}{\rho}\right)\right| \\
\leq & \theta_{i} \frac{\|z\|}{|\rho|}|\rho-1| \\
& +\left|\xi_{i}\left(\frac{z}{\rho}\right)-F_{i}\left(\frac{z}{\rho}\right)\right| \\
\leq & \theta_{i} \alpha|\rho-1|+N_{i} .
\end{aligned}
$$

This is the process of the proof for Lemma 6.

Remark 7. From the analysis of Lemma 6, we know that the approximate accuracy of (8) can be deduced by employing (7) and the time-varying parameter $\rho$, which reveals that the accuracy of approximation can be guaranteed by the timevarying $\rho$ and the Lipschitz coefficient of unknown functions.

Assumption 8. There exist $n$ positive unknown constants $N_{k}$ and fuzzy logic systems such as the form of (7) such that $\sup _{z \in \widetilde{U}}\left|\Delta_{i}-F_{i}(z)\right| \leq N_{i}$. 
If Assumption 8 is true, then we introduce the symbols $F(z)=\left[F_{1}^{T}(z), \ldots, F_{n}^{T}(z)\right]^{T}$, and we can easily get inequality $\sup _{z \in \widetilde{U}}\|\Delta(z)-F(z)\| \leq \sqrt{\sum_{i=1}^{n} N_{i}^{2}} \triangleq N$. From Assumption 2, we define $\sup _{z \in \widetilde{U}}\left\|\left(l_{1}, \ldots, l_{n}\right)\right\| \leq \sqrt{\sum_{i=1}^{n} l_{i}^{2}} \triangleq L$. For brevity, we denote $\widehat{N}=\widehat{N}(t)$ and $\widehat{L}=\widehat{L}(t)$ to represent the estimation values of $N$ and $L$, respectively, and let $\widetilde{N}=\widehat{N}-N$ and $\widetilde{L}=$ $\widehat{L}-L$ represent the estimate errors.

Control Objective. In this paper, a fuzzy adaptive controller is designed to make the output of robot manipulator system (1) track reference model (3) in finite time. Meanwhile, all the signals can be ensured to be bounded in the desired closedloop system.

\section{Fuzzy Adaptation Algorithms Control Design}

In the light of the control target, the following designed scheme fuzzy adaptive switching controller is given:

$$
u= \begin{cases}F(x), & \|e\|>\alpha|\rho|, \\ u_{a}+u_{b}, & \|e\| \leq \alpha|\rho|,\end{cases}
$$

where $u_{a}=M(q) K e+v, v=-B^{T} P e M_{\max }^{-1}\left[\|G\|+\|C\|+\tau_{s}+\right.$ $\left.\tau_{d}\right] /\left\|e^{T} P B\right\| M_{\min }^{-1}$, and $u_{b}=F(x / \rho)$.

$$
\begin{gathered}
\dot{\rho}= \begin{cases}\frac{1}{2 \alpha^{2} \rho}\left(\lambda+\pi_{1}\right), & \|e\|>\alpha|\rho| \\
-\frac{1}{2 \sigma_{1}} \rho-\frac{2}{\gamma} \alpha\|P B\| \pi_{2}, & \|e\| \leq \alpha|\rho|,\end{cases} \\
\dot{\vec{L}}= \begin{cases}0, & \|e\|>\alpha|\rho| \\
-\frac{1}{\sigma_{2}} \widehat{L}+\frac{2}{\delta} \alpha|\rho|\|P B\| \pi_{3}, & \|e\| \leq \alpha|\rho|,\end{cases}
\end{gathered}
$$

$$
\begin{aligned}
& \dot{\hat{N}} \\
& = \begin{cases}\frac{2}{\mu}\|e\| \cdot\|B\| \cdot\left\|M^{-1}(q)\right\|, & \|e\|>\alpha|\rho| \\
-\frac{1}{\sigma_{3}} \widehat{N}+\frac{2 \alpha}{\beta}|\rho|\|P B\|\left\|M^{-1}(q)\right\|, & \|e\| \leq \alpha|\rho|,\end{cases}
\end{aligned}
$$

where $\pi_{1}=2\left\|A_{m}\right\| \cdot\|e\|^{2}+2\|e\| \cdot\|B\|[(\|\bar{P} q\|+\|\bar{Q} \dot{q}\|+$ $\left.\left.\left\|B_{1} r\right\|\right)+M_{\max }^{-1}\left(\widehat{N}+\|C(q, \dot{q})\| \cdot\|\dot{q}\|+\|G(q)\|+\left\|F_{s}(q)\right\|+\left\|T_{d}\right\|\right)\right]$, $\pi_{2}=\left\|M^{-1}(q)\right\|\left[\left(\alpha+\left(\left\|x_{m}\right\| /|\rho|\right)|1-\rho|\right) \widehat{L}+\widehat{N}+\left\|B_{1} r\right\|+\right.$ $\|\bar{P} q\|+\|\bar{Q} \dot{q}\| \widetilde{\operatorname{sign}}(\rho), \widetilde{\operatorname{sign}(\rho)}=\{1, \rho>0 ;-1, \rho \leq 0\}$, $\pi_{3}=\left\|M^{-1}(q)\right\|\left(\alpha+\left(\left\|x_{m}\right\| / \rho\right)|1-\rho|\right)$. The parameters $\alpha, \sigma_{1}$, $\sigma_{2}, \lambda, \gamma, \delta$, and $\beta$ are the given positive constants.
Theorem 9. Consider the robot manipulators (1) and reference model (3) with Assumptions 1-8. The controller (11) with the adaptation algorithms (12) can be synchronized between the velocity and acceleration system (1) with reference model (3). At the same time, the parameter $\rho$ and estimate values $\widehat{N}$ and $\widehat{L}$ all can be ensured to be uniformly ultimately bounded.

Proof. The procedure of the proof of Theorem 9 includes the following two cases.

Case $1(\|e\|>\alpha|\rho|)$. With this condition, we make $s=$ $s(e, \rho, \widetilde{\mathcal{E}}, \widetilde{L})=\|e\|^{2}-\alpha^{2} \rho^{2}+(1 / 2) \eta \widetilde{L}^{2}+(1 / 2) \mu \widetilde{N}^{2}$. It is clear that $s>0$; we define the positive function $\bar{V}=(1 / 2) s^{2}$, by using controller (12) with the case of Lipschitz coefficient under Assumption 2, and then the derivative of $\bar{V}$ which relates to $t$ along the error dynamical system (4) can be obtained as

$$
\begin{aligned}
\dot{\bar{V}} & =s\left(\dot{e}^{T} e+e^{T} \dot{e}-2 \alpha^{2} \rho \dot{\rho}+\eta \dot{\hat{L}} \widetilde{L}+\mu \dot{\hat{N}} \widetilde{N}\right) s\left\{2 e^{T} A_{m}^{T} e\right. \\
& +2 e^{T} B M^{-1}(q)(\tau-\Delta)-2 e^{T} B\left(\bar{P} q+\bar{Q} \dot{q}+B_{1} r\right) \\
& -2 e^{T} B M^{-1}(q)\left[C(q, \dot{q}) \dot{q}+G(q)+F_{s}(q)+T_{d}\right] \\
& \left.-2 \alpha^{2} \rho \dot{\rho}+\eta \dot{\hat{L}} \widetilde{L}+\mu \dot{\hat{N}} \widetilde{N}\right\} \leq s\left\{2\left\|A_{m}\right\| \cdot\|e\|^{2}\right. \\
& +2\|e\| \cdot\|B\|\left(\|\bar{P} q\|+\|\bar{Q} \dot{q}\|+\left\|B_{1} r\right\|\right)+2\|e\| \cdot\|B\| \\
& \cdot M_{\max }^{-1}\left[\widehat{N}+\|C(q, \dot{q})\| \cdot\|\dot{q}\|+\|G(q)\|+\left\|F_{s}(q)\right\|\right. \\
& \left.+\left\|T_{d}\right\|\right]-2 \alpha^{2} \rho \dot{\rho}+\mu \dot{\hat{N}} \widetilde{N}-2\|e\| \cdot\|B\| M_{\max }^{-1}(q) \widetilde{N} \\
& +\eta \dot{\hat{L}} \widetilde{L}\}=-\lambda s .
\end{aligned}
$$

According to [38], (13) implies that the extended state vector $E=\left(e^{T}, \rho, \widehat{L}^{T}, \widehat{N}^{T}\right)^{T}$ can reach the sliding surface $s=0$ in a limited period of times.

Case $2(\|e\| \leq \alpha|\rho|)$. We choose the candidate Lyapunov function $V=e^{T} P e+(1 / 2) \gamma \rho^{2}+(1 / 2) \delta \widetilde{L}^{2}+(1 / 2) \beta \widetilde{N}^{2}$. If Assumptions 1-8 are all satisfied, then the derivative of $V(t)$ following the error dynamical system (4) is given by

$$
\begin{aligned}
\dot{V} & =e^{T}\left(A_{m}^{T} P+P A_{m}\right) e+2 e^{T} P B h(q, \dot{q}, \tau, r)+\gamma \rho \dot{\rho} \\
& +\delta \dot{\dot{L}} \widetilde{L}+\beta \dot{\widehat{N}} \widetilde{N}=-e^{T} Q e+2 e^{T} P B\{v \\
& -M^{-1}(q)\left[C(q, \dot{q}) \dot{q}+G(q)+F_{s}(q)+T_{d}\right] \\
& \left.+M^{-1}(q)\left[u_{b}-\Delta(q, \dot{q})\right]-B_{1} r-\bar{P} q-\bar{Q} \dot{q}\right\}+\gamma \rho \dot{\rho} \\
& +\delta \dot{\hat{L}} \widetilde{L}+\beta \dot{\hat{N}} \widetilde{N} .
\end{aligned}
$$

By using controller (11), we can obtain that

$$
\begin{aligned}
& 2 e^{T} P B\left\{v-M^{-1}(q)\left[C(q, \dot{q}) \dot{q}+G(q)+F_{s}(q)+T_{d}\right]\right\} \\
& \quad= \begin{cases}-2 e^{T} P B \frac{B^{T} P e M_{\max }^{-1}\left(\|C(q, \dot{q})\|+\|G(q)\|+\tau_{s}+\tau_{d}\right)}{\left\|e^{T} P B\right\| M_{\min }^{-1}}, & e^{T} P B \neq 0 \\
0, & e^{T} P B=0,\end{cases}
\end{aligned}
$$




$$
\begin{aligned}
- & 2 e^{T} P B M^{-1}(q)\left[C(q, \dot{q}) \dot{q}+G(q)+F_{s}(q)+T_{d}\right] \leq 2 \frac{\left\|e^{T} P B\right\|\left(\|C(q, \dot{q})\|+\|G(q)\|+\tau_{s}+\tau_{d}\right) M_{\max }^{-1}}{M_{\min }^{-1}} \\
& -2\left\|e^{T} P B\right\|\left[\|C(q, \dot{q}) \dot{q}\|+\|G(q)\|+\tau_{s}+\tau_{d}\right] M^{-1}(q) \\
& \leq 2\left\|e^{T} P B\right\|\left[\|C(q, \dot{q}) \dot{q}\|+\|G(q)\|+\tau_{s}+\tau_{d}\right]\left(1-\frac{M_{\max }^{-1}}{M_{\min }^{-1}}\right) \leq 0 .
\end{aligned}
$$

Since $e=x-x_{m}$ and the inequality $\|x\|-\left\|x_{m}\right\| \leq\left\|x-x_{m}\right\| \leq$ $\alpha|\rho|$ is known, then we have the following inequality:

$$
\|x\| \leq\left\|x-x_{m}\right\|+\left\|x_{m}\right\| \leq \alpha|\rho|+\left\|x_{m}\right\| .
$$

From Lemma 6 and controller (11), we can obtain

$$
\begin{aligned}
\dot{V} \leq & -e^{T} Q e+2 e^{T} P B M^{-1}(q)\left\{\left[u_{b}-\Delta(q, \dot{q})\right]-B_{1} r\right. \\
& -\bar{P} q-\bar{Q} \dot{q}\}+\gamma \rho \dot{\rho}+\delta \dot{\hat{L}} \widetilde{L}+\beta \dot{\hat{N}} \widetilde{N} \leq-e^{T} Q e \\
& +2 \alpha|\rho|\|P B\| \cdot\left\|M^{-1}(q)\right\|\left[L\left(\alpha+\frac{\left\|x_{m}\right\|}{|\rho|}|1-\rho|\right)\right. \\
& \left.+N+\left\|B_{1} r\right\|+\|\bar{P} q\|+\|\bar{Q} \dot{q}\|\right]+\gamma \rho \dot{\rho}+\delta \dot{\hat{L}} \widetilde{L} \\
& +\beta \dot{\hat{N}} \widetilde{N}=-e^{T} Q e+2 \alpha|\rho|\|P B\| \cdot\left\|M^{-1}(q)\right\| \\
& \cdot\left[\widehat{L}\left(\alpha+\frac{\left\|x_{m}\right\|}{|\rho|}|1-\rho|\right)+\widehat{N}+\left\|B_{1} r\right\|+\|\bar{P} q\|\right. \\
& +\|\bar{Q} \dot{q}\|]+\gamma \rho \dot{\rho}+\delta \dot{\hat{L}} \widetilde{L}-2 \alpha|\rho|\|P B\| \cdot\left\|M^{-1}(q)\right\| \\
& +\left(\alpha+\frac{\left\|x_{m}\right\|}{|\rho|}|1-\rho|\right) \widetilde{L}+\beta \dot{\hat{N}} \widetilde{N}-2 \alpha|\rho| \cdot\|P B\| \\
& \cdot\left\|M^{-1}(q)\right\| \widetilde{N} .
\end{aligned}
$$

In terms of adaptive laws (12), the inequality holds as follows:

$$
\dot{V} \leq-\lambda_{\min }(Q)\|e\|^{2}-\frac{\gamma}{2 \sigma_{1}} \rho^{2}-\frac{\delta}{\sigma_{2}} \widehat{L} \widetilde{L}-\frac{\beta}{\sigma_{3}} \widehat{N} \widetilde{N} .
$$

This is because the following inequality is true:

$$
\begin{aligned}
-\frac{\delta}{\sigma_{2}} \widehat{L} \widetilde{L} & =-\frac{\delta}{\sigma_{2}} \widetilde{L}^{2}-\frac{\delta}{\sigma_{2}} L \widetilde{L} \leq-\frac{\delta}{\sigma_{2}} \widetilde{L}^{2}+\frac{\delta}{2 \sigma_{2}} \widetilde{L}^{2}+\frac{\delta}{2 \sigma_{2}} L^{2} \\
& \leq-\frac{\delta}{2 \sigma_{2}} \widetilde{L}^{2}+\frac{\delta}{2 \sigma_{2}} L^{2} .
\end{aligned}
$$

Similarly, we have

$$
-\frac{\beta}{\sigma_{3}} \widehat{N} \widetilde{N} \leq-\frac{\beta}{2 \sigma_{3}} \widetilde{N}^{2}+\frac{\beta}{2 \sigma_{3}} N^{2} .
$$

Substituting (19) and (20) into (18), it follows that

$$
\begin{aligned}
\dot{V} \leq & -\lambda_{\min }(Q)\|e\|^{2}-\frac{\gamma}{2 \sigma_{1}} \rho^{2}-\frac{\delta}{2 \sigma_{2}} \widetilde{L}^{2}-\frac{\beta}{2 \sigma_{3}} \widetilde{N}^{2} \\
& +\frac{\delta}{2 \sigma_{2}} L^{2}+\frac{\beta}{2 \sigma_{3}} N^{2} .
\end{aligned}
$$

Let $\omega=\min \left\{\lambda_{\min }(Q) / \lambda_{\max }(P), \gamma / \sigma_{1}, \delta / \sigma_{2}, \beta / \sigma_{3}\right\}$ and $\phi=$ $\left(\delta / 2 \sigma_{2}\right) L^{2}+\left(\beta / 2 \sigma_{3}\right) N^{2}$; then (21) becomes

$$
\dot{V} \leq-\omega V(t)+\phi
$$

and multiplying $e^{\omega t}$ on both sides in the above inequality we have

$$
\frac{d}{d t}\left\{V(t) e^{\varpi t}\right\} \leq \phi e^{\omega t}
$$

integrating both sides of (23) over $[0, t]$, the following inequality holds:

$$
0 \leq V(t) \leq\left[V(0)-\frac{\phi}{\omega}\right] e^{-\omega t}+\frac{\phi}{\omega} .
$$

The above inequality means that all signals in the closed-loop systems are uniformly ultimately bounded, so the state $E=$ $\left(e^{T}, \rho, \widehat{L}^{T}, \widehat{N}^{T}\right)^{T}$ of error dynamical system (4) is bounded. This is the whole proof of Theorem 9 .

\section{Simulation Example}

To illustrate the control design method in this section, the dynamic equations given as follows with a two-link manipulator are studied:

$$
\begin{aligned}
& {\left[\begin{array}{cc}
\bar{\alpha}_{11}\left(q_{2}\right) & \bar{\alpha}_{12}\left(q_{2}\right) \\
\bar{\alpha}_{21}\left(q_{2}\right) & \bar{\alpha}_{22}
\end{array}\right]\left[\begin{array}{l}
\ddot{q}_{1} \\
\ddot{q}_{2}
\end{array}\right]} \\
& =\left[\begin{array}{c}
\bar{\beta}_{12}\left(q_{2}\right) \dot{q}_{1}^{2}+2 \bar{\beta}_{12}\left(q_{2}\right) \dot{q}_{1} \dot{q}_{2} \\
-\bar{\beta}_{12}\left(q_{2}\right) \dot{q}_{2}^{2}
\end{array}\right] \\
& +\left[\begin{array}{c}
\bar{\gamma}_{1}\left(q_{1}, q_{2}\right) g \\
\bar{\gamma}_{2}\left(q_{1}, q_{2}\right) g
\end{array}\right]-F_{d} \dot{q}-F_{s}+T_{d}+\left[\begin{array}{l}
\tau_{1} \\
\tau_{2}
\end{array}\right],
\end{aligned}
$$

where $\bar{\alpha}_{11}\left(q_{2}\right)=\left(m_{1}+m_{2}\right) r_{1}^{2}+m_{2} r_{2}^{2}+2 m_{2} r_{1} r_{2} \cos \left(q_{2}\right)+J_{1}$, $\bar{\alpha}_{12}\left(q_{2}\right)=\bar{\alpha}_{21}\left(q_{2}\right)=m_{2} r_{2}^{2}+m_{2} r_{1} r_{2} \cos \left(q_{2}\right), \bar{\alpha}_{22}=m_{2} r_{2}^{2}+J_{2}$, 


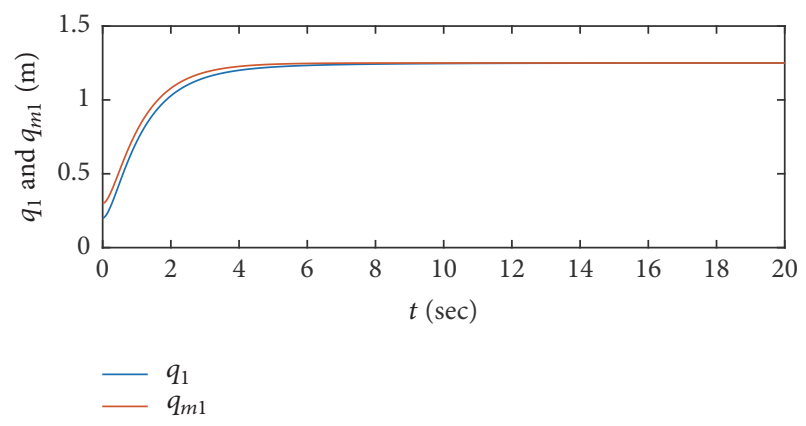

(a)

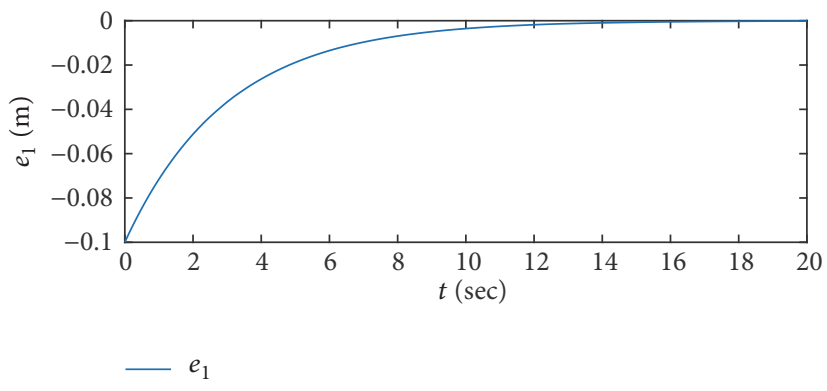

(b)

Figure 1: (a) The output of tracking of joint 1. (b) The output of tracking error of joint 1.

$\bar{\beta}_{12}\left(q_{2}\right)=m_{2} r_{1} r_{2} \sin \left(q_{2}\right), \bar{\gamma}_{1}\left(q_{1}, q_{2}\right)=-\left[\left(m_{1}+m_{2}\right) r_{1} \cos \left(q_{2}\right)+\right.$ $\left.m_{2} r_{2} \cos \left(q_{1}+q_{2}\right)\right], \bar{\gamma}_{2}\left(q_{1}, q_{2}\right)=-m_{2} r_{2} \cos \left(q_{1}+q_{2}\right), F_{d}=$ $\left[\begin{array}{ll}5 \cos \left(\dot{q}_{1}\right) & \sin \left(q_{1}\right) q_{2} \\ \sin \left(q_{2}\right) q_{1} & 3 \cos \left(\dot{q}_{2}\right)\end{array}\right], F_{s}=\left[\begin{array}{l}1.8 \operatorname{sgn}\left(\dot{q}_{1}\right) \\ 1.2 \operatorname{sgn}\left(\dot{q}_{2}\right)\end{array}\right]$, and $T_{d}=\left[\begin{array}{l}0.8 \cos \left(q_{2}\right) \\ 1.5 \sin \left(q_{1}\right)\end{array}\right]$. We know $\tau_{\mathrm{g}}=10, \tau_{s}=1.8$, and $\tau_{d}=1.5$. The parameters in (25) are given as $g=9.8 \mathrm{~m} / \mathrm{s}, r_{1}=1 \mathrm{~m}, r_{2}=0.8 \mathrm{~m}, J_{1}=5 \mathrm{~kg} \cdot \mathrm{m}^{2}$, $J_{2}=5 \mathrm{~kg} \cdot \mathrm{m}^{2}, m_{1}=0.5 \mathrm{~kg}$, and $m_{2}=1.5 \mathrm{~kg}$. The reference model is given by

$$
\dot{x}_{m}=A_{m} x_{m}+B_{m} r(t)
$$

in which $A_{m}=\left[\begin{array}{cccc}0 & 0 & 1 & 0 \\ 0 & 0 & 0 & 1 \\ -4 & 0 & -5 & 0 \\ 0 & -4 & 0 & -5\end{array}\right], B_{m}=\left[\begin{array}{ll}0 & 0 \\ 0 & 0 \\ 1 & 0 \\ 0 & 1\end{array}\right], r(t)=\left[\begin{array}{l}5 \\ 5\end{array}\right]$, $t>0$, and the initial conditions of system (25) and (26) are selected as $x(0)=(0.2,2,0,0)^{T}$ and $x_{m}(0)=(0.3,1.9,0,0)^{T}$, respectively. Initial values of update laws are chosen as $\rho(0)=$ $0.5, \widehat{L}(0)=0.3$, and $\widehat{N}(0)=0.7$, and the parameters in the simulation are chosen as $\eta=3, \mu=1, \lambda=0.1, \alpha=20, \gamma=2$, $\delta=5, \beta=6, \sigma_{1}=0.001, \sigma_{2}=0.002$, and $\sigma_{3}=0.003$. We assume that $F_{d} \dot{q}=\Delta(q, \dot{q})=\Delta\left(x_{1}, x_{2}, x_{3}, x_{4}\right)$ is unknown. We choose $\widetilde{U}=\widetilde{U}_{1} \times \widetilde{U}_{2} \times \widetilde{U}_{3} \times \widetilde{U}_{4}=[-20,20] \times[-20,20] \times$ $[-20,20] \times[-20,20]$, and the membership functions are chosen as $\mu_{N B}\left(x_{i}\right)=1 /\left(1+e^{\left(x_{i}+20\right)}\right), \mu_{N M}\left(x_{i}\right)=1 /\left(1+e^{\left(x_{i}+10\right)}\right)$, $\mu_{N S}\left(x_{i}\right)=1 /\left(1+e^{\left(x_{i}+0.1\right)}\right), \mu_{P S}\left(x_{i}\right)=1 /\left(1+e^{\left(x_{i}-0.1\right)}\right), \mu_{P M}\left(x_{i}\right)=$ $1 /\left(1+e^{\left(x_{i}-10\right)}\right)$, and $\mu_{P B}\left(x_{i}\right)=1 /\left(1+e^{\left(x_{i}-20\right)}\right)$. The FLS (fuzzy logic system) $F_{1}(q)$ is comprised of selecting 6 fuzzy rules $A_{1}^{l} \times A_{2}^{l} \times A_{3}^{l} \times A_{4}^{l} \rightarrow B^{l}(l=1,2, \ldots, 6)$ to represent $\Delta_{1}(q)=$ $5 \cos \left(\dot{q}_{1}\right) \dot{q}_{1}+\sin \left(q_{1}\right) q_{2} \dot{q}_{2}=5 \cos \left(\dot{x}_{1}\right) \dot{x}_{1}+\sin \left(x_{1}\right) x_{2} \dot{x}_{2}$.

If $x_{1}$ is $N B, x_{2}$ is $N M, \dot{x}_{1}$ is $P S$, and $\dot{x}_{2}$ is $P B$,

then $\Delta_{1}$ is $N B$.

If $x_{1}$ is $N M, x_{2}$ is $N S, \dot{x}_{1}$ is $P S$, and $\dot{x}_{2}$ is $P M$, then $\Delta_{1}$ is $N M$.

If $x_{1}$ is $P S, x_{2}$ is $N M, \dot{x}_{1}$ is $P B$, and $\dot{x}_{2}$ is $N M$, then $\Delta_{1}$ is NS.

If $x_{1}$ is $P M, x_{2}$ is $P B, \dot{x}_{1}$ is $N S$, and $\dot{x}_{2}$ is $N B$, then $\Delta_{1}$ is $P S$.

If $x_{1}$ is $P B, x_{2}$ is $P S, \dot{x}_{1}$ is $N B$, and $\dot{x}_{2}$ is $N M$,

then $\Delta_{1}$ is $P M$.

If $x_{1}$ is $N S, x_{2}$ is $P B, \dot{x}_{1}$ is $N M, \dot{x}_{2}$ is $N S$,

then $\Delta_{1}$ is $P B$.
Similarly, the FLS $F_{2}(q)$ is constituted by following 6 fuzzy rules $\bar{A}_{1}^{l} \times \bar{A}_{2}^{l} \times \bar{A}_{3}^{l} \times \bar{A}_{4}^{l} \rightarrow \bar{B}^{l}$ to represent $\Delta_{2}(q)=$ $\sin \left(q_{2}\right) q_{1} \dot{q}_{1}+3 \cos \left(\dot{q}_{2}\right) \dot{q}_{2}=\sin \left(x_{2}\right) x_{1} \dot{x}_{1}+3 \cos \left(\dot{x}_{2}\right) \dot{x}_{2}$ as follows:

If $x_{1}$ is $N B, x_{2}$ is $P B, \dot{x}_{1}$ is $P S$, and $\dot{x}_{2}$ is $N S$, then $\Delta_{2}$ is $P B$.

If $x_{1}$ is $P M, x_{2}$ is $N S, \dot{x}_{1}$ is $P B$, and $\dot{x}_{2}$ is $N M$, then $\Delta_{1}$ is $N M$.

If $x_{1}$ is $N S, x_{2}$ is $P M, \dot{x}_{1}$ is $N B$, and $\dot{x}_{2}$ is $P B$, then $\Delta_{1}$ is $P M$.

If $x_{1}$ is $P B, x_{2}$ is $P M, \dot{x}_{1}$ is $N S$, and $\dot{x}_{2}$ is $N B$, then $\Delta_{1}$ is $P S$.

If $x_{1}$ is $P S, x_{2}$ is $P B, \dot{x}_{1}$ is $N B$, and $\dot{x}_{2}$ is $P M$, then $\Delta_{1}$ is NS.

If $x_{1}$ is $N S, x_{2}$ is $P S, \dot{x}_{1}$ is $P B$, and $\dot{x}_{2}$ is $N B$, then $\Delta_{1}$ is $N B$.

Figures 1,2, and 3 show the simulation results of the two-link manipulators with fuzzy adaptive controller scheme, respectively.

Figures 1 and 2 show the output and tracking errors between system (25) and references signals in (26), by use of the fuzzy adaptive control in the paper; the adaptive laws about time-varying parameter, approximation errors, and unknown Lipschitz coefficients laws converge to zero in Figure 3, and good tracking performance is achieved.

\section{Conclusions}

This paper develops a novel adaptation algorithm fuzzy controller for robot manipulators with uncertain nonlinearity terms; the fuzzy logic systems with parameters are used to compensate for the unknown nonlinear terms in the dynamical robot manipulators. From the analysis and the simulation results, we know that no matter what types of FLSs are used, the approximation accuracies of FLSs can be estimated automatically by online learning, making the number of adaptation algorithms have no relation to the structure of original FLSs; this new design idea can make the person who uses his/her experience focus on high interpretability and neglecting the adaptive parameters to 


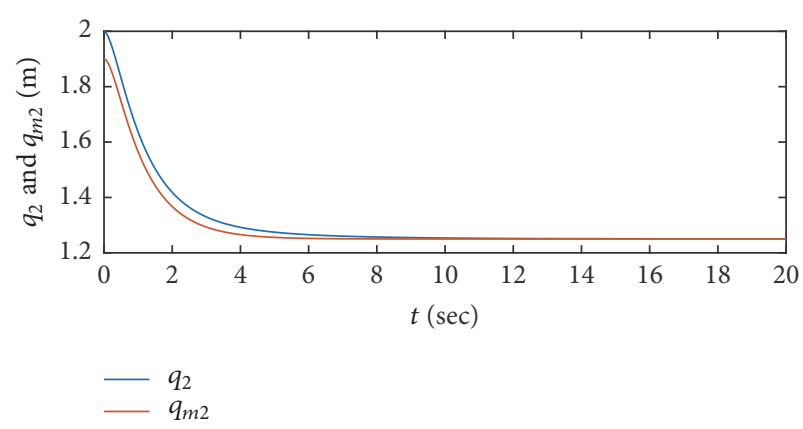

(a)

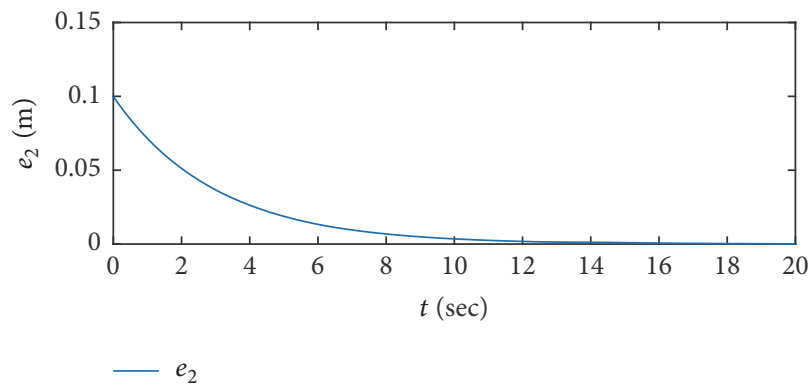

(b)

FIgURE 2: (a) The output of tracking of joint 2. (b) The output of tracking error of joint 2.

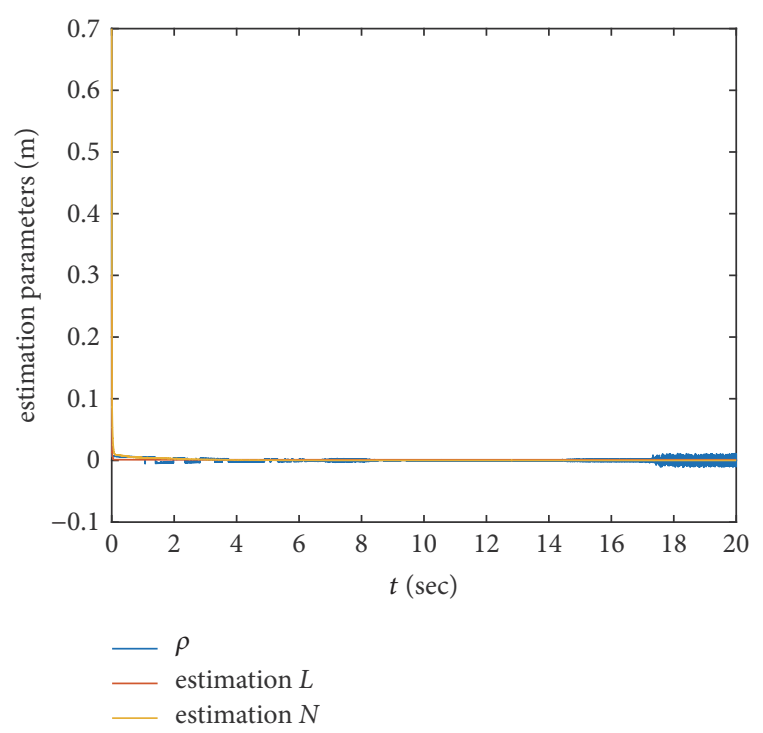

FIGURE 3: The time response of estimation of parameters.

synthesize fuzzy controller. The final simulation example has shown the effectiveness of the design method in this paper.

\section{Conflicts of Interest}

The authors declare that they have no conflicts of interest.

\section{Acknowledgments}

This work was funded by the financial support from CSC (China Scholarship Council) Program (201708615095), National Natural Science Foundation of China (51405387), Shaanxi Province Key Research and Development Plan General Project of Agriculture (2017NY-129), Shaanxi Natural Science Foundation (2016JQ5051), and Scientific Research Plan Projects of Shaanxi Education Department (17JK0709).

\section{References}

[1] S. Ullrich and T. Kuhlen, "Haptic palpation for medical simulation in virtual environments," IEEE Transactions on Visualization and Computer Graphics, vol. 18, no. 4, pp. 617-625, 2012.
[2] L. M. Di Diodato, R. Mraz, S. N. Baker, and S. J. Graham, "A haptic force feedback device for virtual reality-fMRI experiments," IEEE Transactions on Neural Systems and Rehabilitation Engineering, vol. 15, no. 4, pp. 570-576, 2007.

[3] S. C. Jacobsen, M. Olivier, F. M. Smith et al., "Research robots for applications in artificial intelligence, teleoperation and entertainment," International Journal of Robotics Research, vol. 23, no. 4-5, pp. 319-330, 2004.

[4] J. Na, G. Herrmann, and K. Zhang, "Improving transient performance of adaptive control via a modified reference model and novel adaption," International Journal of Robust and Nonlinear Control, vol. 27, no. 8, pp. 1351-1372, 2017.

[5] C. Yang, C. Zeng, P. Liang, Z. Li, R. Li, and C.-Y. Su, "Interface Design of a Physical Human-Robot Interaction System for Human Impedance Adaptive Skill Transfer," IEEE Transactions on Automation Science and Engineering, vol. 15, no. 1, pp. 329340, 2018.

[6] C. Yang, J. Luo, Y. Pan, Z. Liu, and C. Su, "Personalized variable gain control with tremor attenuation for robot teleoperation," IEEE Transactions on Systems, Man, and Cybernetics: Systems, pp. 1-12, 2017.

[7] M. A. Al-Mouhamed, M. Nazeeruddin, and N. Merah, "Design and instrumentation of force feedback in telerobotics," IEEE Transactions on Instrumentation and Measurement, vol. 58, no. 6, pp. 1949-1957, 2009.

[8] X. Yang, C.-C. Hua, J. Yan, and X.-P. Guan, "A New MasterSlave Torque Design for Teleoperation System by T-S Fuzzy Approach," IEEE Transactions on Control Systems Technology, vol. 23, no. 4, pp. 1611-1619, 2015.

[9] C. Yang, X. Wang, Z. Li, Y. Li, and C. Su, "Teleoperation control based on combination of wave variable and neural networks," IEEE Transactions on Systems, Man, and Cybernetics: Systems, vol. 47, no. 8, pp. 2125-2136, 2017.

[10] H. Nijmeijer and I. M. Mareels, "An observer looks at synchronization," IEEE Transactions on Circuits and Systems I: Fundamental Theory and Applications, vol. 44, no. 10, pp. 882890, 1997.

[11] A. Lora, E. Panteley, and A. Zavala-Ro, "Adaptive observers with persistency of excitation for synchronization of chaotic systems," IEEE Transactions on Circuits and Systems I: Regular Papers, vol. 56, no. 12, pp. 2703-2716, 2009.

[12] C. Yang, H. Wu, Z. Li, W. He, N. Wang, and C. Su, "Mind Control of A Robotic Arm with Visual Fusion Technology," IEEE Transactions on Industrial Informatics, p. 1, 2017.

[13] J. Taheri-Kalani and M. J. Khosrowjerdi, "Adaptive trajectory tracking control of wheeled mobile robots with disturbance 
observer," International Journal of Adaptive Control and Signal Processing, vol. 28, no. 1, pp. 14-27, 2014.

[14] F. Hong, S. S. Ge, B. Ren, and T. H. Lee, "Robust adaptive control for a class of uncertain strict-feedback nonlinear systems," International Journal of Robust and Nonlinear Control, vol. 19, no. 7, pp. 746-767, 2009.

[15] Y. Huang, J. Na, X. Wu, and G. Gao, "Approximation-Free Control for Vehicle Active Suspensions with Hydraulic Actuator," IEEE Transactions on Industrial Electronics, p. 1, 2018.

[16] J. Na, M. N. Mahyuddin, G. Herrmann, X. Ren, and P. Barber, "Robust adaptive finite-time parameter estimation and control for robotic systems," International Journal of Robust and Nonlinear Control, vol. 25, no. 16, pp. 3045-3071, 2015.

[17] S. Wang, X. Ren, J. Na, and T. Zeng, "Extended-state-observerbased funnel control for nonlinear servomechanisms with prescribed tracking performance," IEEE Transactions on Automation Science and Engineering, vol. 14, no. 1, pp. 98-108, 2017.

[18] J. Na, A. S. Chen, G. Herrmann, R. Burke, and C. Brace, "Vehicle Engine Torque Estimation via Unknown Input Observer and Adaptive Parameter Estimation," IEEE Transactions on Vehicular Technology, vol. 67, no. 1, pp. 409-422, 2018.

[19] L. Cheng, Z.-G. Hou, M. Tan, Y. Lin, and W. Zhang, "Neuralnetwork-based adaptive leader-following control for multiagent systems with uncertainties," IEEE Transactions on Neural Networks and Learning Systems, vol. 21, no. 8, pp. 1351-1358, 2010.

[20] Y.-J. Liu, S. Lu, and S. Tong, "Neural Network Controller Design for an Uncertain Robot with Time-Varying Output Constraint," IEEE Transactions on Systems, Man, and Cybernetics: Systems, vol. 47, no. 8, pp. 2060-2068, 2017.

[21] Z. Li, H. Xiao, C. Yang, and Y. Zhao, "Model predictive control of nonholonomic chained systems using general projection neural networks optimization," IEEE Transactions on Systems, Man, and Cybernetics: Systems, vol. 45, no. 10, pp. 1313-1321, 2015.

[22] C. Yang, X. Wang, L. Cheng, and H. Ma, "Neural-learningbased telerobot control with guaranteed performance," IEEE Transactions on Cybernetics, vol. 47, no. 10, pp. 3148-3159, 2017.

[23] J. Na, Q. Chen, X. Ren, and Y. Guo, "Adaptive prescribed performance motion control of servo mechanisms with friction compensation," IEEE Transactions on Industrial Electronics, vol. 61, no. 1, pp. 486-494, 2014.

[24] Z. Liu, F. Wang, and Y. Zhang, "Adaptive Visual Tracking Control for Manipulator with Actuator Fuzzy Dead-Zone Constraint and Unmodeled Dynamic," IEEE Transactions on Systems, Man, and Cybernetics: Systems, vol. 45, no. 10, pp. 13011312, 2015.

[25] Z. Li, C. Yang, C.-Y. Su, S. Deng, F. Sun, and W. Zhang, "Decentralized Fuzzy Control of Multiple Cooperating Robotic Manipulators with Impedance Interaction," IEEE Transactions on Fuzzy Systems, vol. 23, no. 4, pp. 1044-1056, 2015.

[26] L. Wang and J. M. Mendel, "Fuzzy basis functions, universal approximation, and orthogonal least-squares learning," IEEE Transactions on Neural Networks and Learning Systems, vol. 3, no. 5, pp. 807-814, 1992.

[27] S. J. Yoo, J. B. Park, and Y. H. Choi, "Adaptive output feedback control of flexible-joint robots using neural networks: Dynamic surface design approach," IEEE Transactions on Neural Networks and Learning Systems, vol. 19, no. 10, pp. 1712-1726, 2008.

[28] F. Sun, Z. Sun, and P.-Y. Woo, "Neural network-based adaptive controller design of robotic manipulators with an observer," IEEE Transactions on Neural Networks and Learning Systems, vol. 12, no. 1, pp. 54-67, 2001.
[29] C. Yang, Y. Jiang, Z. Li, W. He, and C.-Y. Su, "Neural control of bimanual robots with guaranteed global stability and motion precision," IEEE Transactions on Industrial Informatics, vol. 13, no. 3, pp. 1162-1171, 2017.

[30] J. Na, Y. Huang, X. Wu, G. Gao, G. Herrmann, and J. Z. Jiang, "Active adaptive estimation and control for vehicle suspensions with prescribed performance," IEEE Transactions on Control Systems Technology, pp. 1-15, 2017.

[31] C. Yang, K. Huang, H. Cheng, Y. Li, and C. Su, "Haptic identification by ELM-controlled uncertain manipulator," IEEE Transactions on Systems, Man, and Cybernetics: Systems, vol. 47, no. 8, pp. 2398-2409, 2017.

[32] W. E. Dixon, "Adaptive regulation of amplitude limited robot manipulators with uncertain kinematics and dynamics," Institute of Electrical and Electronics Engineers Transactions on Automatic Control, vol. 52, no. 3, pp. 488-493, 2007.

[33] Y.-J. Liu, Y. Gao, S. Tong, and Y. Li, "Fuzzy approximation-based adaptive backstepping optimal control for a class of nonlinear discrete-time systems with dead-zone," IEEE Transactions on Fuzzy Systems, vol. 24, no. 1, pp. 16-28, 2015.

[34] H. Wang, Z. Wang, Y.-J. Liu, and S. Tong, "Fuzzy tracking adaptive control of discrete-time switched nonlinear systems," Fuzzy Sets and Systems, vol. 316, pp. 35-48, 2017.

[35] B. M. Novakovic, "Fuzzy logic robot control synthesis without any rule base," in Proceedings of the 8th International Conference on Advanced Robotics, ICAR'97, pp. 141-146, 1997.

[36] B. M. Novakovic, "Fuzzy logic control synthesis without any rule base," IEEE Transactions on Systems, Man, and Cybernetics, Part B: Cybernetics, vol. 29, no. 3, pp. 459-466, 1999.

[37] M. Zhihong, A. P. Paplinski, and H. R. Wu, "A robust MIMO terminal sliding mode control scheme for rigid robotic manipulators," Institute of Electrical and Electronics Engineers Transactions on Automatic Control, vol. 39, no. 12, pp. 2464-2469, 1994.

[38] J. J. E. Slotine and W. Li, Applied Nonlinear Control, Prentice Hall, Englewood Cliffs, NJ, USA, 1991. 


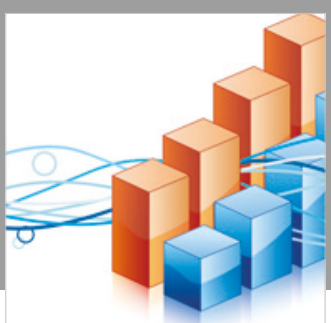

Advances in

Operations Research

\section{-n-m}
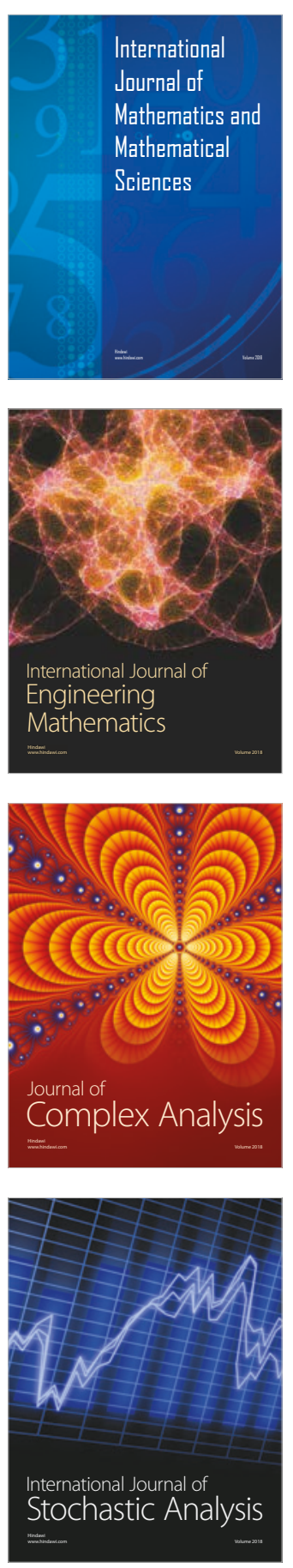
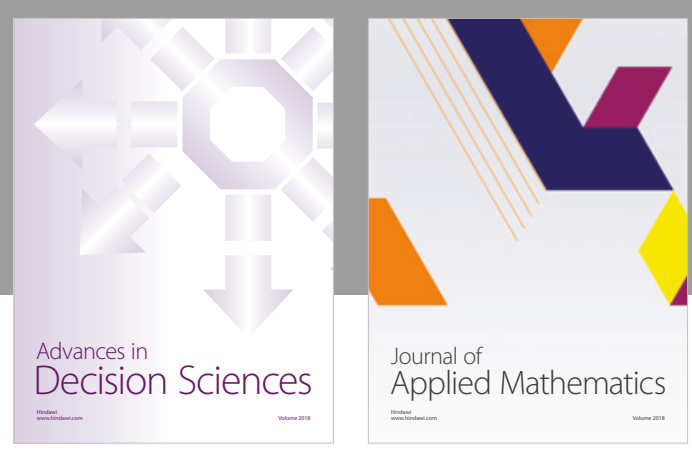

Journal of

Applied Mathematics
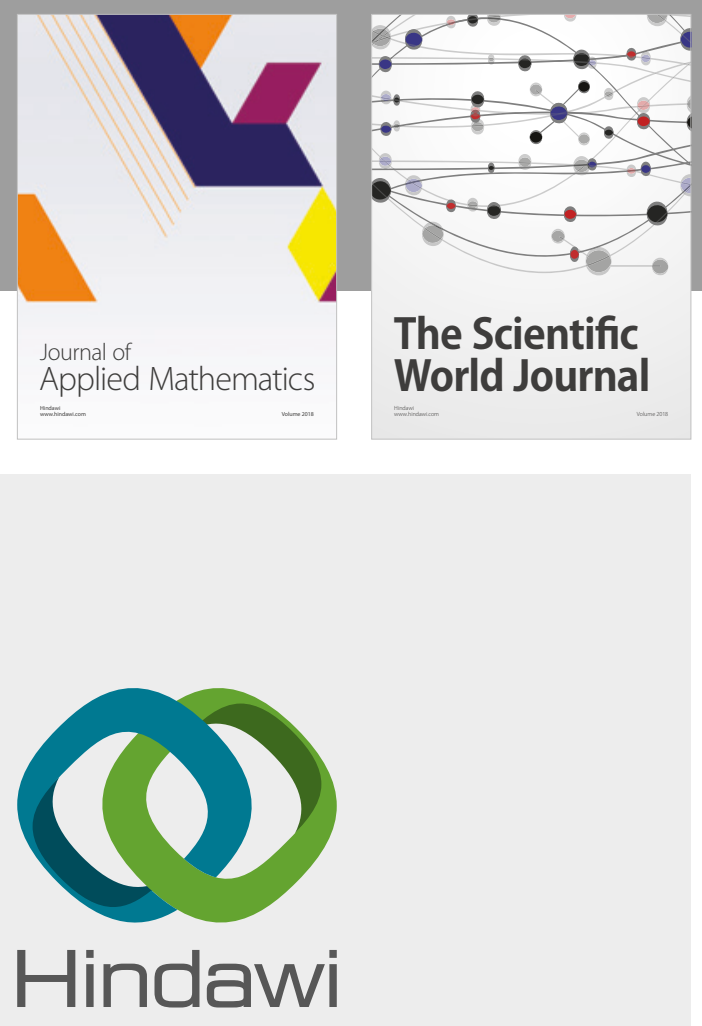

Submit your manuscripts at

www.hindawi.com

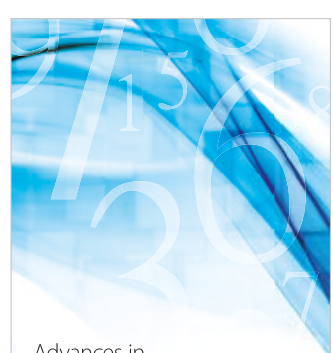

Advances in
Numerical Analysis
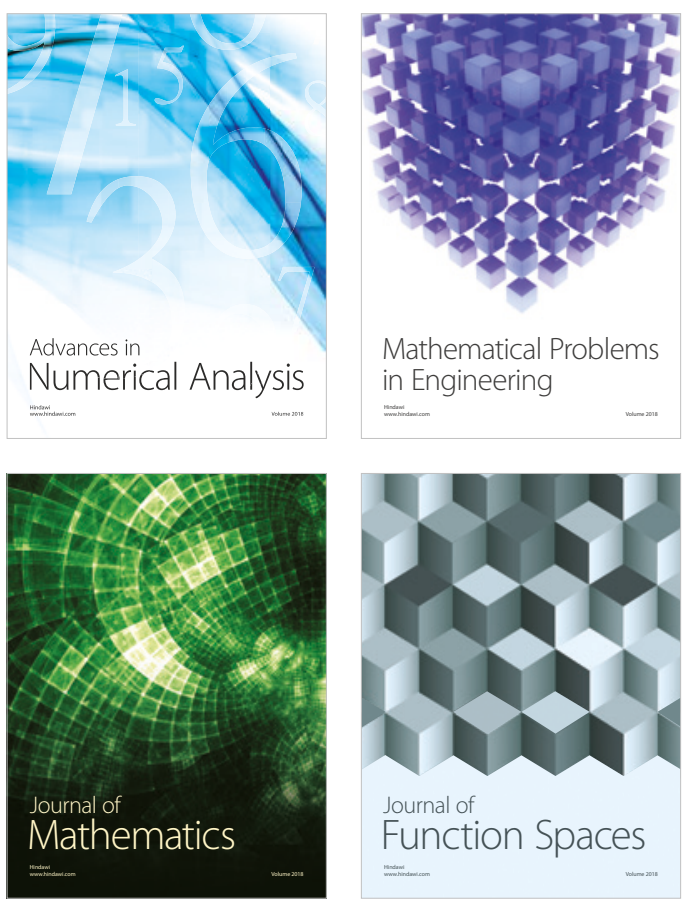

Mathematical Problems in Engineering

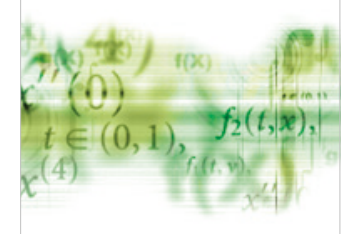

International Journal of

Differential Equations

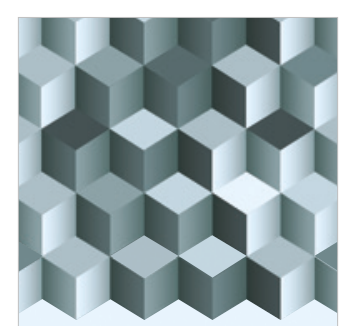

Journal of

Function Spaces
The Scientific

World Journal

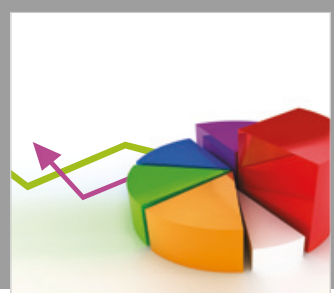

Journal of

Probability and Statistics
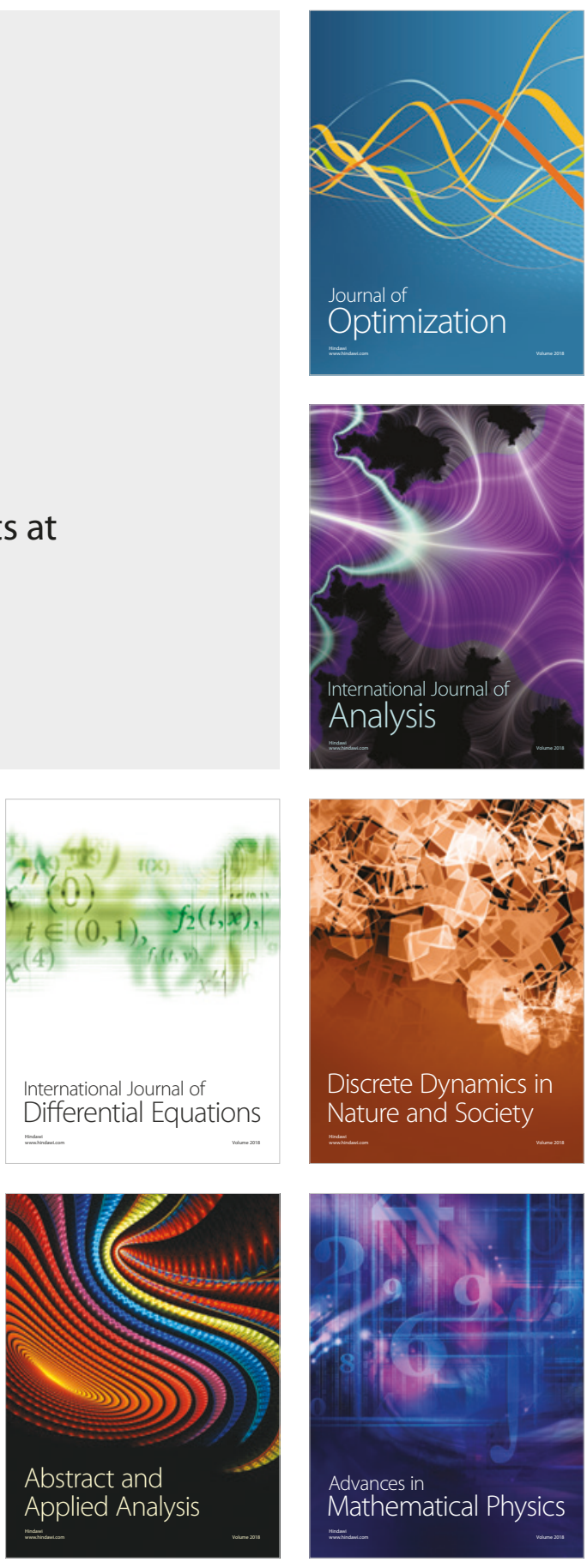